Gilbert Buti, Daniel Faget, Olivier Raveux et Solène Rivoal (dir.), Moissonner la mer. Économies, sociétés et pratiques halieutiques méditerranéennes (XVe-XXI siècle)

Paris/Aix-en-Provence, Éditions Karthala/MMSH, 2018

\title{
Romain Grancher
}

\section{OpenEdition}

\section{Journals}

Édition électronique

URL : http://journals.openedition.org/artefact/4323

DOI : 10.4000/artefact.4323

ISSN : 2606-9245

Éditeur :

Association Artefact. Techniques histoire et sciences humaines, Presses universitaires du Midi

Édition imprimée

Date de publication : 15 octobre 2019

Pagination : 237-243

ISBN : 978-2-8107-0650-1

ISSN : 2273-0753

\section{Référence électronique}

Romain Grancher, « Gilbert Buti, Daniel Faget, Olivier Raveux et Solène Rivoal (dir.), Moissonner la mer. Économies, sociétés et pratiques halieutiques méditerranéennes ( $x v^{e}$-xxle siècle) », Artefact [En ligne], 10 | 2019, mis en ligne le 06 août 2020, consulté le 28 novembre 2020. URL : http://

journals.openedition.org/artefact/4323; DOI : https://doi.org/10.4000/artefact.4323

Ce document a été généré automatiquement le 28 novembre 2020.

\section{cc)}

Artefact, Techniques, histoire et sciences humaines est mise à disposition selon les termes de la Licence Creative Commons Attribution - Pas d'Utilisation Commerciale - Pas de Modification 4.0 International. 


\section{Gilbert Buti, Daniel Faget, Olivier Raveux et Solène Rivoal (dir.),} Moissonner la mer. Économies, sociétés et pratiques halieutiques méditerranéennes ( $\mathrm{XV}^{e}-\mathrm{XXI} I^{e}$ siècle)

Paris/Aix-en-Provence, Éditions Karthala/MMSH, 2018

\section{Romain Grancher}

\section{RÉFÉRENCE}

Gilbert Buti, Daniel Faget, Olivier Raveux et Solène Rivoal (dir.), Moissonner la mer. Économies, sociétés et pratiques halieutiques méditerranéennes ( $\mathrm{XV}^{e}$-XXI e siècle), Paris/Aix-enProvence, Éditions Karthala/MMSH, 2018, 395 p.

1 Fruit d'un colloque international à la croisée de l'histoire, de l'anthropologie et des sciences de l'environnement, l'ouvrage dirigé par Gilbert Buti, Daniel Faget, Olivier Raveux et Solène Rivoal présente, à travers une vingtaine d'études de cas de qualité inégale, un panorama des recherches consacrées au monde de la pêche méditerranéen entre le $\mathrm{xv}^{\mathrm{e}}$ et le $\mathrm{xxI}^{\mathrm{e}}$ siècle. Centré exclusivement sur la partie occidentale du bassin, et plus particulièrement sur ses eaux espagnoles, françaises et italiennes, il propose « une lecture critique de l'histoire des communautés halieutiques" plaçant l'accent sur les « mécanismes anciens qui ont pu conduire à la situation de crise actuelle » (p. 11-13). On retrouve ici l'une des perspectives esquissées dans le champ de la marine environmental history par des historiens comme Jeffrey Bolster, Poul Holm ou Bo Poulsen par exemple, dont on aurait pu s'attendre à voir les travaux cités, sinon discutés ${ }^{1}$. Caractérisée par une baisse inquiétante des captures imputée dès l'introduction à une surexploitation de longue durée des ressources marines, cette situation de crise 
n'engage pas seulement les acteurs de la profession, mais aussi les historiennes et les historiens, les halieutes ou les biologistes selon les auteurs de l'ouvrage, qui appellent de leurs vœux une coopération plus étroite entre le monde de la pêche et le monde de la recherche. Dans cette perspective, leur ambition première est d'inviter la communauté scientifique à opérer, par-delà les barrières disciplinaires, un retour sur les expériences halieutiques du passé, dans l'idée que certaines d'entre elles pourraient inspirer une nécessaire réorientation des politiques de gestion actuelles. Toutefois, il s'agit surtout d'une louable déclaration d'intention car malgré « la volonté partagée par l'ensemble des auteurs de cette publication de bâtir des ponts interdisciplinaires " (p.14), le résultat final est d'une facture somme toute classique, les articles réunis s'organisant davantage autour de thématiques communes aux différents terrains explorés par les contributrices et les contributeurs qu'à partir d'une approche transversale éprouvée collectivement.

2 Pour autant, l'ouvrage apporte un éclairage intéressant sur la variété et les transformations des manières de "moissonner la mer " méditerranéenne et, plus largement, sur les sociétés littorales structurées par l'activité de pêche côtière. Pratique d'activation des ressources marines, la pêche implique de considérer non seulement les savoirs et les techniques permettant la capture du poisson, mais également les régimes d'accès à la mer, la construction juridique et sociale des territoires côtiers, les formes d'organisation du travail maritime, le fonctionnement des marchés des produits de la mer, ou encore les modes de régulation de l'environnement marin - autant d'objets de recherche qui viennent tisser des fils à l'intérieur de ce recueil d'articles et lui conférer une cohérence d'ensemble en dépit de l'hétérogénéité des pratiques halieutiques analysées. De la multiplicité des communautés techniques locales, dont le particularisme est d'abord d'ordre lexical comme le soulignent aussi bien Ambra Zambernardi que Sylviane Llinares et Gilbert Buti dans leurs contributions respectives, émerge ainsi progressivement l'image d'un monde de la pêche méditerranéen façonné par des expériences communes.

3 Au-delà des phénomènes de circulation bien mis en évidence par Maurizio Gangemi dans son article sur le nomadisme halieutique des communautés de pêcheurs des Pouilles, on repère en effet, d'une contribution à l'autre, des processus à l'œuvre relativement similaires, qui confèrent au cadre méditerranéen une certaine unité, même si une ouverture sur d'autres espaces géographiques aurait permis d'en mieux cerner l'originalité et, sans doute, de la relativiser. C'est tout d'abord un monde en mutation que donne à voir cet ouvrage collectif, dont l'un des apports les plus importants tient à l'analyse des processus de transfert et d'acclimatation des nouvelles techniques de pêche tout au long de la période envisagée. De ce point de vue, l'article d'Alfons Garrido et Marcel Pujol qui ouvre le volume vient d'emblée inscrire cette problématique dans la longue durée, en montrant comment l'introduction de la thonaire au XIV siècle, puis du sardinal au XVI ${ }^{e}$ siècle, est venue reconfigurer en profondeur les régimes halieutiques préexistant sur les côtes de la Catalogne et ouvrir ainsi la voie à des pêches commerciales pratiquées de plus en plus loin du rivage et intégrées à des marchés de plus en plus connectés aux villes côtières et à leurs arrièrepays. D'une manière générale, on assiste en effet au cours de la période moderne et contemporaine à un élargissement progressif des horizons halieutiques, dont témoignent bien les cas adriatiques étudiés par Solène Rivoal ou Maria Lucia de Nicolò. Cette domestication technique de la mer, qui voit les pêcheurs quitter le rivage pour 
exploiter successivement la zone côtière puis la haute-mer, s'exerce également en direction des abysses, comme le rappellent Daniel Faget et Olivier Raveux dans leur article consacré à l'exploitation du corail, un produit encore largement ignoré par l'historiographie. Retraçant dans un premier temps les multiples innovations techniques apportées depuis le $\mathrm{x}^{\mathrm{e}}$ siècle à la croix de Saint-André, l'outil longtemps privilégié par les corailleurs, ils montrent ensuite comment la conquête du milieu sousmarin s'accélère au $\mathrm{XIX}^{\mathrm{e}}$ siècle avec l'invention du scaphandre, qui permet alors une véritable mise en coupe réglée des fonds coralliens de la Méditerranée.

4 Toutefois, l'acclimatation de nouvelles techniques ne s'accompagne pas forcément de l'abandon des pratiques de pêche en usage antérieurement, notamment de celles qui reposent sur l'exploitation de la mer depuis le rivage ou à proximité des côtes grâce à des installations dites «fixes" ou «sédentaires». Ainsi, tant les trabucchi de l'Adriatique décrits par Ezio Ritrovato que les madragues observées par Ambra Zambernardi à l'occasion d'une riche enquête ethnographique en Sardaigne sont restés en usage jusqu'à nos jours et il faut donc envisager l'évolution des pratiques halieutiques, non comme un processus de transition d'une technique à l'autre, mais plutôt comme un processus de diversification et de densification, impliquant une exploitation multiforme de l'ensemble du territoire maritime par différentes catégories de pêcheurs définis par les "métiers » ou les «arts » qu'ils exercent. Une chose est sûre, c'est que ce processus contribue à redéfinir les régimes de la territorialité maritime et qu'il engendre de multiples conflits, d'autant plus que les communautés littorales ont tendance à revendiquer un droit d'usage exclusif sur ce qu'on pourrait appeler des «terroirs » ou des " communs » halieutiques - c'est peut-être là d'ailleurs une réelle spécificité méditerranéenne qui aurait mérité en tout cas d'être soulignée et discutée ${ }^{2}$. Sur ce point, l'article d'Alida Clemente offre des perspectives stimulantes en analysant les débuts de la pisciculture industrielle en Italie à travers la question de l'enclôture de la mer, mais, d'une manière générale, on peut tout de même s'étonner que la littérature économique et surtout anthropologique consacrée à la construction juridique et sociale des territoires de pêche ne soit jamais convoquée (à l'exception de The Tragedy of the Commons de Garrett Hardin...), alors qu'elle aurait sans doute permis de mieux problématiser bon nombre de conflits inter ou intracommunautaires autour de l'accès à la ressource ${ }^{3}$.

De fait, la défense des territoires communautaires et des formes d'organisation locale de la pêche face à l'intrusion de pêcheurs étrangers ou face à l'introduction de nouvelles pratiques constitue l'une des clés permettant d'expliquer la résurgence dans les sources de la thématique du «dépeuplement de la mer » (p.186), repérée par les auteurs de nombreuses contributions. Mobilisée tout au long de la période pour dénoncer le caractère destructeur de certaines techniques de pêche (en particulier des " arts traînants »), cette rhétorique de l'épuisement de la ressource frappe par son ubiquité, mais soulève néanmoins un double problème qui n'est pas directement affronté: d'une part, celui de la réalité et, le cas échéant, de l'ampleur de ces phénomènes d'épuisement, certes difficiles à démontrer positivement pour des périodes où les données fiables font défaut; et, d'autre part, celui de leur imputation à des pratiques de pêche trop intensives. Faut-il, en somme, croire les acteurs historiques lorsqu'ils évoquent l'amenuisement de la ressource et l'attribuent à sa surexploitation ? Eux-mêmes, pourrait-on se demander à la suite de Paul Veyne, y croyaient-ils vraiment? Peut-on enfin s'appuyer seulement sur des mentions «anecdotiques» de 
pénurie de poissons pour retracer l'histoire écologique des populations marines ${ }^{4}$ ? $\mathrm{Ce}$ sont là des questions complexes, qui n'ont cessé de diviser le champ de la marine environmental history depuis les années 1990, et auxquelles les différents auteurs de ce volume n'apportent pas de réponse étayée ni univoque - même si la plupart s'accordent toutefois pour prendre les acteurs au sérieux et semblent ainsi accréditer, à raison sans doute, l'idée selon laquelle les racines économiques et technologiques du mal environnemental seraient anciennes.

Monde en mutation, monde en conflit et, finalement, monde en crise : face à cela, les acteurs institutionnels et sociaux directement concernés, au premier rang desquels les États riverains de la Méditerranée, ont cherché à apporter des réponses et des remèdes ou, à défaut, des palliatifs. Plusieurs articles détaillent ainsi l'instauration de véritables politiques annonaires. Dans le cas de Gênes, Paolo Calcagno montre par exemple que c'est en faisant jouer le ressort fiscal que les édiles ont tenté de sécuriser et d'accroître les approvisionnements en produits de la mer au cours du XVIII siècle. Solène Rivoal analyse quant à elle très finement le cas d'un véritable projet d'expansion du territoire halieutique vénitien au-delà de la lagune, en direction des eaux dalmates, considérées alors comme sous-exploitées et donc riches de potentialités économiques. Toutefois, c'est seulement à partir du $\mathrm{xIX}^{\mathrm{e}}$ siècle que s'élaborent de véritables politiques d'encouragement et de développement de la pêche. Ainsi, dans l'Algérie du premier $\mathrm{xx}^{\mathrm{e}}$ siècle étudiée par Hugo Vermeren, la métropole s'appuie sur les scientifiques de la station locale d'aquiculture et de pêche pour favoriser l'emploi des populations kabyles dans le secteur de l'industrie halieutique. Dans l'Italie post-unification, Alida Clemente montre pour sa part l'émergence d'une utopie industrielle qui se traduit par des initiatives privées largement soutenues par l'État, visant à rationaliser l'exploitation des ressources halieutiques par la mise en culture de la mer. Enfin, de manière parallèle, se sont progressivement mises en place des politiques de conservation, dans le but explicite de limiter l'impact des activités humaines sur les populations marines. Les pratiques contemporaines de gestion de la ressource présentées par Laurence Le Diréach et al. ou par Mohammed Ramdani et al. s'inscrivent en effet dans une longue histoire de la régulation environnementale, comme le montrent bien Gilbert Buti et Sylviane Llinares en analysant les enjeux de la mission d'inspection des pêches confiée au commissaire Chardon à la veille de la Révolution : outil de gouvernement destiné à la constitution d'une expertise administrative en matière halieutique, l'enquête est également une occasion pour élaborer, sur le terrain même, des mesures de conservation négociées avec les acteurs du monde de la pêche.

7 Ce dernier point ramène finalement à la question des rapports entre praticiens de la mer et praticiens des sciences de la mer soulevée dans l'introduction de cet ouvrage et, plus généralement, à l'opportunité de se tourner vers le passé des sociétés halieutiques pour y chercher des modes d'exploitation plus vertueux et des formes d'organisation plus démocratiques. Si la démarche est en soi légitime et pourrait sûrement s'avérer intéressante, elle doit cependant se garder de tout romantisme et ne pas conduire à idéaliser des communautés qui, comme le rappelle Daniel Faget (p.12) et comme le montrent bien la plupart des contributions au volume, sont traversées par des conflits et des contradictions profondes. Aussi est-il permis d'émettre de sérieuses réserves lorsque les prud'homies de pêcheurs par exemple sont présentées comme "un modèle de gestion de "communs" qui a fait ses preuves dans l'histoire " (Élisabeth Tempier, p. 243) ou comme un «modèle » égalitaire de « régulation économique et écologique 
des pêches» (Yannick Bosc, p. 221) - cela, non pas pour reconduire un argument de la tragédie des communs dont la pauvreté théorique et les soubassements idéologiques ne sont plus à démontrer ${ }^{5}$, mais simplement pour souligner, dans le sillage même de cet ouvrage, la complexité économique, sociale et juridique des collectifs façonnés par la pratique de la pêche.

\section{BIBLIOGRAPHIE}

ACHESON James M., The Lobster Gangs of Maine, University Press of New England, Hanover, 1988. ARMIERO Marco, « La risorsa contesta : norme, conflitti e technologie tra i pescatori meridionali (XIX sec.) », Meridiana, $\mathrm{n}^{\circ}$ 31, 1998, p. 179-206.

BARNARD Michaela, HOLM Poul et STARKEY David J. (dir.), Oceans past. Management insights from the History of Marine Animal Populations, Earthscan, Londres/Sterling, 2008.

BERKES Fikret, "Common Property Resource Management and Cree Indian Fisheries in Subarctic Canada ", dans MCCAY Bonnie J. et ACHESON James M., The Question of the Commons, University of Arizona Press, Tucson, 1987, p. 66-91.

BOLSTER W. Jeffrey, « Opportunities in Marine Environmental History », Environmental History, vol. 11, n 3, 2006, p. 567-597.

BOLSTER W. Jeffrey, The Mortal Sea. Fishing the Atlantic in the Age of Sail, The Belknap Press of Harvard University Press, Cambridge/London, 2012.

DURREMBERGER E. Paul et PÁLSSON Gísli, « Ownership at sea: fishing territories and access to sea resources ", American Anthropologist, vol. 14, nº 3, 1987, p. 508-522.

INGOLD Alice, « Les sociétés d'irrigation : bien commun et action collective », Entreprises et histoire, $\mathrm{n}^{\circ} 50,2008 / 1$, p. $19-35$.

LOCHER Fabien, « Les pâturages de la guerre froide : Garrett Hardin et la "tragédie des communs" ", Revue d'histoire moderne et contemporaine, $\mathrm{n}^{\circ}$ 60, 2013/1, p. 7-36.

MCCAY Bonnie J., « Systems Ecology, People Ecology, and the Anthropology of Fishing Communities », Human Ecology, vol. 6, n 4, 1978, p. 397-422.

OSTROM Elinor, Governing the Commons: The Evolution of Institutions for Collective Action, Cambridge University Press, Cambridge, 1990.

PAUly Daniel, « Anecdotes and the Shifting Baseline Syndrome of Fisheries », Trends in Ecology and Evolution, vol. 10, $\mathrm{n}^{\circ}$ 10, 1995, p. 430.

SCHWERDTNER MÁÑEZ Kathleen et POULSEN Bo (dir.), Perspectives on Oceans Past. A Handbook of Marine Environmental History, Springer Science+Business Media, Dordrecht, 2016.

SCHLAGER Edella et OSTROM Elinor, « Property-Rights Regimes and Natural Resources: A Conceptual Analysis ", Land Economics, vol. 68, n 3, 1992, p. 249-262. 


\section{NOTES}

1. Barnard, Holm et starkey 2008 ; Bolster, 2006 ; Bolster, 2012 ; schwerdtner Máñez et Poulsen, 2016.

2. En repartant par exemple de ARMIERO, 1998.

3. Mccay, 1978 ; Durremberger et Pálsson, 1987 ; Berkes, 1987 ; Acheson, 1988 ; ostrom, 1990 ; schlager et ostrom, 1992.

4. Pauly, 1995.

5. Ingold, 2008 ; Locher, 2013.

\section{AUTEURS}

\section{ROMAIN GRANCHER}

Chargé de recherche CNRS, Laboratoire TEMOS (FRE 2015) 